\title{
ELECTRIC COUNTERSHOCK AND COLD STRESS EFFECTS ON LIVER AND ADRENAL GLAND
}

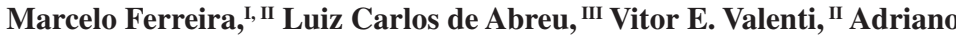

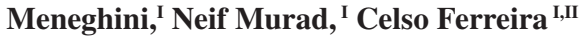

doi: $10.1590 / \mathrm{S} 1807-59322010000300009$

Ferreira M, de Abreu LC, Valenti VE, Meneghini A, Murad N, Ferreira C. Effects of electrical countershock and cold stress on the liver and adrenal gland. Clinics. 2010;65(3):291-6.

OBJECTIVES: Cold exposure induces glycogen and lipid depletion in the liver and the adrenal gland, respectively. However, no previous study has determined the effects of electrical countershock on those tissues. We aimed to evaluate the effects of electrical countershock on lipid depletion in the adrenal gland and on glycogen depletion in the liver.

METHODS: We used 40 male Wistar rats divided into four groups: the control group, in which the animals were subjected to a resting period of seven days; the electrical discharge group, in which the animals were subjected to a resting period followed by administration of ten 300-mV electrical discharges; the electrical post-discharge group, in which the animals received ten electrical shocks $(300 \mathrm{mV})$ followed by rest for seven consecutive days; and the cold stress group, in which the animals were subjected to a resting period and were then exposed to $-8^{\circ} \mathrm{C}$ temperatures for four hours. All animals underwent a laparotomy after treatment. The lipid and glycogen depletions are presented using intensity levels (where $+=$ low intensity and $++++=$ high intensity, with intermediate levels in between).

RESULTS: The rats exposed to the cold stress presented the highest glycogen and lipid depletion in the liver and the adrenal gland, respectively. Furthermore, we noted that the electrical countershock significantly increased lipid depletion in the adrenal gland and glycogen depletion in the liver. One week after the electrical countershock, the liver and adrenal gland profiles were similar to that of the control group.

CONCLUSION: Electrical countershock immediately increased the glycogen depletion in the liver and the lipid depletion in the adrenal gland of rats.

KEYWORDS: Electrical Countershock; Cold; Stress; Rats; Hypothermia.

\section{INTRODUCTION}

More than 20 years ago, Selye ${ }^{1}$ recognized that the physiological system is activated by stress and that protecting and restoring the body can also damage the system. ${ }^{2,3}$ Exposure to low temperatures is considered to be an important stressing physical agent. ${ }^{4,5}$ Previous studies

\footnotetext{
${ }^{\text {I }}$ Departamento de Clínica Médica, Disciplina de Cardiologia, Faculdade de Medicina do ABC - Santo André/SP, Brazil.

II Departamento de Medicina, Disciplina de Cardiologia, Universidade Federal de São Paulo (UNIFESP) - São Paulo/SP, Brazil.

III Departamento de Morfologia e Fisiologia, Faculdade de Medicina do ABC - Santo André/SP, Brazil.

Email: celsoferreira.dmed@epm.br

Tel.: 55114993.5403

Received for publication on August 31, 2009

Accepted for publication on November 26, 2009
}

have reported that acute exposure to cold stress can damage heart tissue ${ }^{6}$ and cause lipid depletion in the adrenal gland and glycogen depletion in the liver, ${ }^{6-8}$ thus suggesting that sympathetic activity and oxidative stress are the sources of these injuries.

Previous studies have demonstrated that cardiomyocytes can be injured by electrical discharges. Under normal circumstances, clinical evaluation following defibrillation has revealed that the electrical energy pulse applied to the thoracic area has enough force to conserve the heart effectively, even though the discharge increases proportionally with patient body weight. ${ }^{9-12}$ Moreover, the magnitude of the damage depends on the electrical pulse waves, and thus, the myocardial injury is highlighted by the unevenness of the ST segment on the electrocardiogram. In addition, a minor aggression was reported when truncated 
biphasic shocks were applied instead of attenuated monophasic sinusoidal discharges. ${ }^{13}$

Although it was recently indicated that cold stress induces glycogen and lipid depletion in the liver and the adrenal gland, respectively, ${ }^{6,14}$ no previous study has investigated the effects of different types of stress on the liver and the adrenal gland. Furthermore, it was previously observed that electrical discharge in rats was able to produce cardiac injury noted through mitochondrial impairment in the heart tissue; ${ }^{9-11}$ therefore, we hypothesized that an electrical discharge would also affect other organs in addition to the heart. In this study, we aimed to evaluate the effects of two different types of stress agents on lipid depletion in the adrenal gland and on glycogen depletion in the liver.

\section{METHODS}

\section{Animals}

In this study, experiments were performed on 40 adult male Wistar rats $(250-350 \mathrm{~g})$. The rats were obtained from the Central Biotery of our university. The temperature was maintained at $22^{\circ} \mathrm{C}$, air humidity was kept at nearly $60 \%$ and the $12 \mathrm{~h}$ light- $12 \mathrm{~h}$ dark cycle was used. The animals had free access to food and water. After an adaptation period of nearly one week, the animals were randomly selected and separated into four groups: the control group $(n=10)$, in which the animals were subjected to a resting period (seven days before experiments) with water and food ad libitum; the electrical discharge group ( $\mathrm{D}, \mathrm{n}=10)$, in which the animals were subjected to a resting period with water and food $a d$ libitum followed by administration of ten $300-\mathrm{mV}$ electrical discharges, in this group lipid and glycogen depletion were analyzed immediately after electrical discharge; the electrical post-discharge group ( $\mathrm{PD}, \mathrm{n}=10$ ), in which the animals received ten electrical discharges $(300 \mathrm{mV})$ and were then given water and food ad libitum for seven consecutive days, in this group lipid and glycogen depletion were analyzed seven days after electrical discharge; and the cold stress group (Cold, $\mathrm{n}=10$ ), in which the animals were subjected to a resting period with water and food ad libitum and were then exposed to $-8^{\circ} \mathrm{C}$ for four hours only once. After the resting period laparotomy was performed in all animals, in order to evaluate lipid and glycogen depletion. Each group contained 10 rats because this number of animals provided sufficient statistical power while minimizing the number of animals used. All procedures were performed in accordance with the ethical guidelines of the National Institutes of Health Guide for the Care and Use of Laboratory Animals, and the study protocol was approved by the Ethical Committee for research at our university.

\section{Induced Hypothermia Procedure}

For the cold stress group, the rats were exposed to cold stress that was induced by placing them in wire mesh cages in an open refrigerated compartment at $-8^{\circ} \mathrm{C}$ for 4 hours. The rats were exposed to this environment only once, and their behavior was observed throughout the stress experiment. ${ }^{7}$ Measurements of the rats' body temperatures confirmed that the rats were able to maintain a body temperature around 37C. The temperature was controlled by the refrigerated compartment. No rats died during the induced hypothermia procedure. $^{6-8}$

\section{Electrical Countershock}

Electrodes adapted for this study $\left(3 \mathrm{M}^{\oplus}\right)$ were positioned on the animal's precordial region to apply the electrical discharges. The electrical discharges were delivered through electrical cardioverter equipment adapted for small rodent animals by the bioengineering department of our university. The equipment was assembled to mimic the equipment used to treat cardiac rhythm reversion disorders in humans. The cardioverter device is able to generate serial voltage impulses of up to $300 \mathrm{mV}$, which corresponds to an energy transmission potential of $3 \mathrm{~J}$. This serial voltage with the combined signal pattern is similar to a capacitor discharge in an RL circuit (inductance and resistance), with a 10 $\mathrm{ms}$ average. The impedance of the developed system for the experimental purpose was not determined due to the complexity and nature of the study methodology. However, the experimental group conditions were always equal, thus allowing a reliable and fair comparison. ${ }^{15}$

\section{Histological Procedure}

All animals were submitted to a laparotomy after an adequate level of ether anesthesia was reached; adequate anesthesia was verified by tail tonus and response to external stimuli (i.e., through the evaluation of vibrissae movements) before and during the surgical procedure. Two pieces of the left lobe of the liver and the right adrenal gland were removed for investigation by light microscopy. The fragments were cut into small pieces of $1 \mathrm{~mm}^{3}$ and were post-fixed in a $1 \% \mathrm{OsO}_{4}$ solution for $2 \mathrm{~h}$; the samples were then dehydrated and embedded in araldite. The silver or gray thin sections $(60-90 \mathrm{~nm})$ were cut using a Porter-Blum MT-B ultramicrotome. The ultra-thin slices were mounted on copper silver grids with 200 patches and were stained with uranyl acetate and lead citrate. The glycogen depletion 
in the hepatocytes and the lipid depletion in the adrenal gland cells were evaluated by three different investigators. Data concerning lipid or glycogen depletion are presented using intensity levels (where $+=$ low intensity and ++++ $=$ high intensity, with levels in between). Each sample was examined by three independent investigators using the same standardized criteria. ${ }^{6-8}$

\section{Statistical Analysis}

To evaluate the data associated with lipid depletion in the adrenal gland cells and with glycogen depletion in the hepatocytes, the Kruskal-Wallis and the Tukey post-hoc tests were applied to compare the independent groups. Agreement of the measurements from the three investigators was evaluated and analyzed using Bartko's intraclass correlation coefficient (see equation below) according to the Fleiss guidelines ${ }^{16}$ (significance set at $\mathrm{p}<0.05$ ).

\section{Bartko's test formula}

$$
R=\frac{N(P M S-E M S)}{N(P M S)+(K-1)(R M S)+(N-1)(K-1) E M S}
$$

$\mathrm{R}=$ Bartko's correlation index; PMS = Patients' Mean

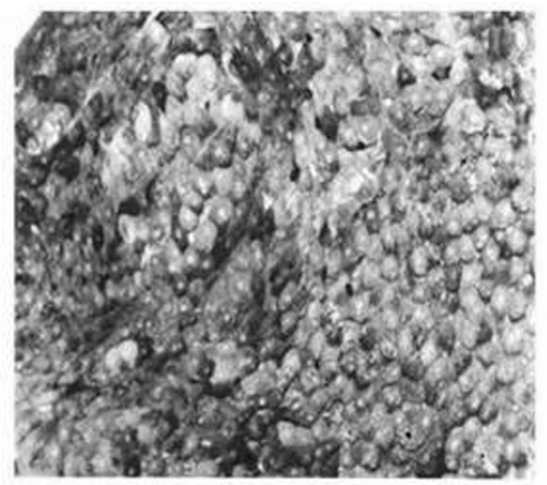

\section{A}

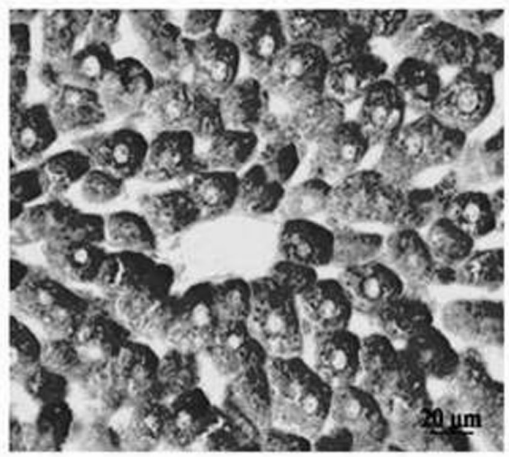

C

Figure 1 - Glycogen depletion in the liver (stained with the PAS method) of the (A) control, (B) discharge, (C) post-discharge and (D) cold stress groups.
Square; RMS = Researcher Mean Square; EMS = Error Mean Square; $\mathrm{N}=$ Number of events; $\mathrm{K}=$ Number of investigators.

\section{RESULTS}

We observed that ten $300-\mathrm{mV}$ electrical shocks altered the glycogen and lipid profiles in the liver (Figure 1 and Table 1) and the adrenal gland (Figure 2 and Table 2 ), respectively. However, one week after the electrical countershock, the liver and lipid characteristics were similar to that of the control group (Figures 1 and 2, Tables 1 and 2).

As hypothesized, the induced hypothermia group presented the highest rate of glycogen depletion in the hepatocytes (Figure 1 and Table 1$)(\mathrm{p}<0.05)$ and the highest rate of lipid depletion in the adrenal gland cortical cells (Figure 2 and Table 2) $(\mathrm{p}<0.05)$. This result indicates that exposure to $-8^{\circ} \mathrm{C}$ temperatures for four hours was an effective stressing agent.

Bartko's correlation index ranged between 0.56-0.95 in all experimental groups, thus validating the experimental methodology. Moreover, differences between the groups' means were statistically significant $(\mathrm{p}<0.05)$.

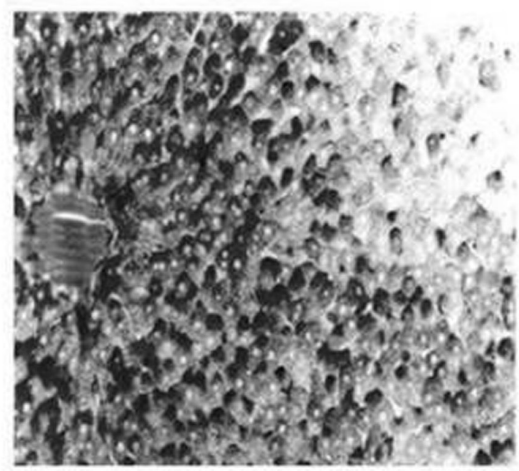

B

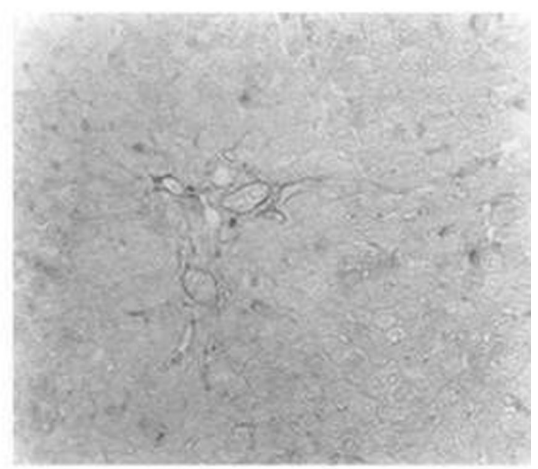

D 


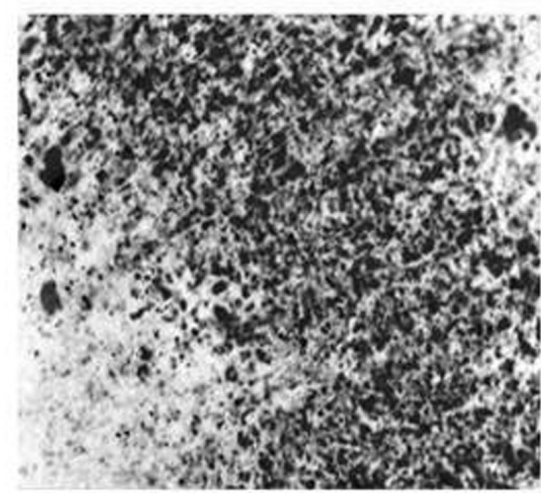

A

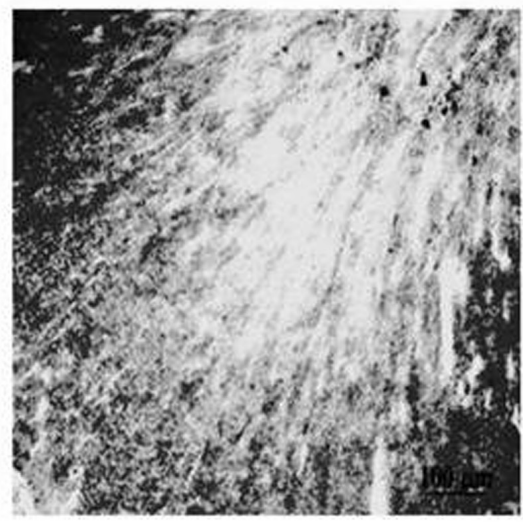

C

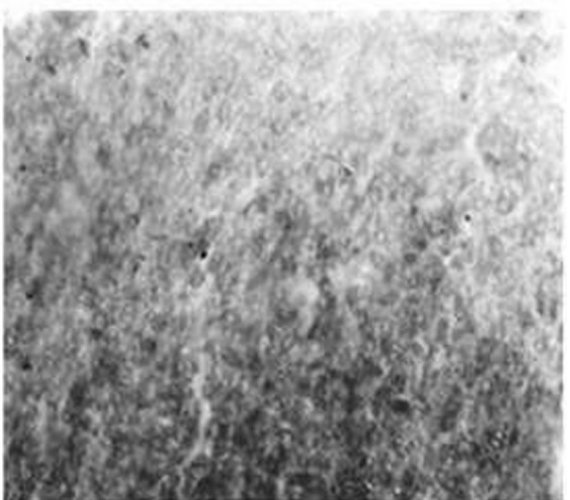

B

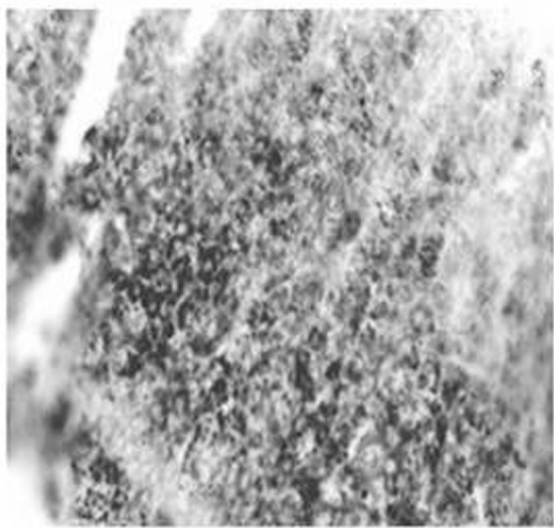

D

Figure 2 - Lipid depletion in the adrenal gland cells (stained with Sudan IV) of the (A) control, (B) discharge, (C) post-discharge and (D) cold stress groups.

Table 1 - Glycogen depletion intensity in the hepatocytes stained with the PAS method. The range is from $+=$ low intensity (higher glycogen depletion) to $++++=$ high intensity (smaller glycogen depletion).

\begin{tabular}{lcccc}
\hline Animal & Control & Discharge & Post-discharge & Cold \\
\hline 1 & ++++ & ++ & ++++ & + \\
2 & ++++ & +++ & ++++ & + \\
3 & +++ & +++ & +++ & ++ \\
4 & +++ & ++ & +++ & + \\
5 & ++++ & ++ & +++ & ++ \\
6 & +++ & ++ & ++++ & ++ \\
7 & ++++ & ++ & ++++ & ++ \\
8 & ++++ & ++ & +++ & + \\
9 & +++ & ++ & ++++ & ++ \\
10 & ++++ & ++ & ++++ & ++ \\
\hline
\end{tabular}

\section{DISCUSSION}

In this study, we investigated the effects of electrical countershock on lipid depletion and glycogen depletion in adrenal gland and liver cells, respectively, in Wistar rats.
Table 2 - Lipid depletion intensity in the adrenal gland cortical cells stained with Sudan IV. The range is from $+=$ low intensity (higher lipid depletion) to $++++=$ high intensity (smaller lipid depletion).

\begin{tabular}{lcccc}
\hline Animal & Control & Discharge & Post-discharge & Stress \\
\hline 1 & ++++ & ++ & ++++ & ++ \\
2 & +++ & ++ & +++ & + \\
3 & +++ & +++ & +++ & ++ \\
4 & ++++ & ++ & +++ & ++ \\
5 & ++++ & ++ & ++++ & ++ \\
6 & ++++ & +++ & +++ & + \\
7 & +++ & ++ & +++ & + \\
8 & ++++ & ++ & ++ & ++ \\
9 & ++++ & +++ & ++++ & + \\
10 & ++++ & ++ & +++ & + \\
\hline
\end{tabular}

Because cold stress increases glycogen depletion in the liver and lipid depletion in the adrenal gland, ${ }^{6,8}$ we compared the intensities of the lipid and glycogen depletions between the cold exposure and the electrical shock groups. Moreover, to investigate if the liver and adrenal gland profiles change over 
time, we also evaluated the lipid and glycogen depletion one week after the electrical shock was applied. As hypothesized, the cold exposure increased the lipid depletion in the adrenal gland and the glycogen depletion in the hepatocytes, in agreement with previous studies. ${ }^{6.8}$ Furthermore, we noted that the electrical discharge immediately reduced the lipid and glycogen intensities in the adrenal gland and liver, respectively.

Behavioral analysis allowed us to observe the stress manifestation in the rats after exposure to $-8^{\circ} \mathrm{C}$ temperatures. Their behavior was similar to the initial reaction proposed by Selye ${ }^{1}$; moreover, Murad et al. ${ }^{17}$ observed similar reactions. In addition, the histological analysis of the liver tissue showed lower concentrations of glycogen in the hepatocytes of the rats exposed to cold stress. Stress conditions cause the release of catecholamine, which in turn causes oxidative glycogenolysis to occur; as a result, glycogenolysis is accelerated, leading to a lower concentration of glycogen in the cortical area of the liver cells. ${ }^{18}$ The histological analysis also demonstrated that the stress group presented a higher depletion of lipids in the cortical area of the adrenal gland cells. This is the result of the release of catecholamine, which stimulates lipolysis in the adrenal gland cells. ${ }^{18}$

According to our findings, electrical countershock immediately impaired the liver and adrenal gland profiles by causing further glycogen and lipid depletion, respectively. To the best of our knowledge, no study regarding this method of analysis has been previously published. As a result, we performed a morphometrical study of the changes in hepatocytes and adrenal gland cells caused by electrical countershock. A previous investigation found that electrical discharges in in vitro chicken cell cultures caused temporary microlesions in the cellular sarcolemma. ${ }^{19}$ Other experiments in dogs have confirmed the effects of direct current discharges on the mitochondria. Many mitochondrial pathological aspects, including loss of membrane integrity, swollen units and disruption, were found even after the application of low-energy endocardial countershocks. ${ }^{20,21}$ Considering the changes shown by electron microscopy on the seventh day in our procedures, two possibilities should be considered: constant effects based on the catecholaminergic action and/or the remission changes derived from the electrical discharges that were caused by the electrical discharge stress condition. A strong hypothesis relating other mechanisms to compensate for the physiologically unviable mitochondria, which were present mainly in the hepatocytes, must also be considered. The mitochondrial damage and the assumed reduction in ATP production may justify the glycogen depletion under the post-cardioversion and post-defibrillation conditions. ${ }^{20}$

The PD (post-electrical discharge) group was used to investigate the delayed liver and adrenal involvement caused by the adrenergic release. At seven days after the experimental procedures, we did not observe any hepatic glycogen or adrenal lipid depletion. Because of the animal adaptation period during the rest stage, the possibilities of dehydration, malnutrition, acid-base balance imbalances and metabolic disorders were eliminated. In addition, due to the short time that elapsed between the discharges and the collection of material for verification, systemic interference due to the catecholamine release is unlikely.

Our study shows that information concerning hepatic glycogen or adrenal lipid depletion should be considered in clinical myocardial protection, both before and after the clinical proceedings associated with transthoracic electrical discharges, particularly in subjects with heart disorders. However, the limitations of the present study were that we did not examine the systolic and diastolic blood pressure, heart rate, contractility, calcium homeostasis, or TnI levels. Additionally, we did not investigate other injury indicators, which could complement the countershock effects on the heart and cardiovascular parameters. We only studied the effects of the electrical discharges on the lipid and glycogen profiles in the adrenal glands and the liver, respectively, as this was the main focus of our research.

In conclusion, our findings showed that electrical countershock immediately depletes glycogen in the liver and lipids in the adrenal gland, similar to the effects of an acute cold stress. These effects were reversed one week after the electrical discharges were applied. Thus, these results show that the electrical discharge procedure induces liver and adrenal gland impairment.

\section{REFERENCES}

1. Selye H. The evolution of the stress concept. Am Sci. 1973;61:692-9.

2. Scorza FA, Colugnati DB, Pansani AP, Sonoda EY, Arida RM, Cavalheiro EA. Preventing tomorrow's sudden cardiac death in epilepsy today: what should physicians know about this? Clinics. 2008;63:38994.
3. Andraus W, Jukemura J, Dutra F, Bechara E, Cunha JE, Leite KR, et al. Oxidative stress is enhanced by hypothermia imposed on ceruleininduced pancreatitis in rats. Clinics. 2007;62:483-90.

4. Gallo Cde B, Mimura MA, Sugaya NN. Psychological stress and recurrent aphthous stomatitis. Clinics. 2009;64:645-8. 
5. Yuksel S, Asma D, Yesilada O. Antioxidative and metabolic responses to extended cold exposure in rats. Acta Biol Hung. 2008;59:57-66.

6. Meneghini A, Ferreira C, Abreu LC, Ferreira M, Fereira Filho C, Valenti VE, et al. Cold stress effects on cardiomyocytes nuclear size in rats: light microscopic evaluation. Rev Bras Cir Cardiovasc. 2008;23:530-3.

7. Meneghini A, Ferreira C, de Abreu LC, Valenti VE, Ferreira M, Filho $\mathrm{CF}$, et al. Memantine prevents cardiomyocytes nuclear size reduction in the left ventricle of rats exposed to cold stress. Clinics. 2009;64:921-6.

8. Bombig MT, Ferreira C, Mora O, Soares JD, Póvoa R, Luna Filho B, et al. Pravastatin protection from cold stress in myocardium of rats. Jpn Heart J. 2003;44:243-55.

9. Ehsani A, Ewy GA, Sobel BE. Effects of electrical countershocks on serum creatine phosphokinase (CPK) isoenzyme activity. Am J Cardiol. 1976;37:12-18.

10. Babbs CF, Tacker WA, Vanvleet JF, Bourland JD, Geddes LA. Therapeutic indices for transchest defibrillator shocks: effective, damaging, and lethal electrical doses. Am Heart J. 1980;99:734-8.

11. Jones JL, Jones RE, Balasky G. Microlesion formation in myocardial cells by high intensity electric field stimulation. Am J Physiol. 1987;253:H480-6.

12. Carvalho VO, Guimarães GV, Ciolac EG, Bocchi EA. Heart rate dynamics during a treadmill cardiopulmonary exercise test in optimized beta-blocked heart failure patients. Clinics. 2008; 63:479-82.

13. Bardy GH, Marchlinski FE, Sharma AD, Worley SJ, Luceri RM, Yee R, et al. Multicenter comparison of truncated biphasic shocks and standard damped sine wave monophasic shocks for transthoracic ventricular defibrillation. Transthoracic Investigators. Circulation. 1996;94:250714.
14. Daud FV, Murad N, Meneghini A, Ferreira M, Ferreira Filho C, Abreu LC, et al. Fluoxetine effects on mitochondrial ultrastructure of right ventricle in rats exposed to cold stress. Rev Bras Cir Cardiovasc. 2009;24:173-9.

15. Ferreira M, Ferreira C, de Abreu LC, Valenti VE, Murad N, Meneghini A, et al. Myocardium tissue changes caused by electrical transthoracic discharges in rats. Int Arch Med. 2009;2:31.

16. Fleiss JL. A critique of recent research on the two-treatment crossover design. Control Clin Trials. 1989;10:237-43.

17. Murad N, Takiuchi K, Lopes AC, Bonilha AM, Souza MM, Demarchi LM, et al. Coenzyme Q10 exogenous administration attenuates cold stress cardiac injury. Jpn Heart J. 2001;42:327-38.

18. Sutherland EW, Robinson GA, Butcher RW. Come aspects of biological role of adenosine 3,5 monophosphate (cyclic AMP). Circulation. 1968;37:279-302.

19. Jones DL, Narayanan N. Defibrillation depresses hear sarcoplasmic reticulum calcium pump: a mechanism of postshock dysfunction. Am J Physiol Heart Circ Physiol. 1998;274:H98-105.

20. Schirmer U, Hemmer W, Lindner KH, Anhäupl T, Wieser T. Ultrastructural alterations in the right and left ventricular myocardium following multiple low energy endocardial countershocks in anesthetized dogs. Pacing Clin Electrophysiol. 1997;20:79-87.

21. Fellows CL, Ahern TS, Chilson DA, Packer DL, Wilber DJ, Mattioni TA, et al. For the transthoracic investigator - Multicenter comparison of truncated biphasic shocks and standard damped sine wave monophasic shocks for transthoracic ventricular defibrillation. Circulation. 1996;94:2507-14. 